(C) [2009] IEEE. Reprinted, with permission, from Wang, Yi., Zhu, Jianguo., Wang, Shuhong., Guo, Youguang \& Xu, Wei. 2009, Nonlinear Magnetic Model of Surface Mounted PM Machines Incorporating Saturation Saliency', IEEE Transactions On Magnetics, Vol. 45, no. 10, pp. 4684-4687. This material is posted here with permission of the IEEE. Such permission of the IEEE does not in any way imply IEEE endorsement of any of the University of Technology, Sydney's products or services. Internal or personal use of this material is permitted. However, permission to reprint/republish this material for advertising or promotional purposes or for creating new collective works for resale or redistribution must be obtained from the IEEE by writing to pubs-permissions@ieee.org. By choosing to view this document, you agree to all provisions of the copyright laws protecting it. 


\title{
Nonlinear Magnetic Model of Surface Mounted PM Machines Incorporating Saturation Saliency
}

\author{
Yi Wang ${ }^{1}$, Jianguo Zhu ${ }^{1}$, Shuhong Wang ${ }^{2}$, Youguang Guo ${ }^{1}$, Wei Xu ${ }^{1}$ \\ ${ }^{1}$ School of Electrical, Mechanical and Mechatronic Systems, University of Technology, Sydney, NSW 2007, Australia \\ ${ }^{2}$ Faculty of Electrical Engineering, Xi'an Jiaotong University, Xi'an 710049, China
}

\begin{abstract}
In this paper a comprehensive nonlinear model of surface mounted permanent magnet synchronous machine (SPMSM) is proposed considering both the structural and the saturation saliencies to enable the numerical simulation of new rotor position detection algorithms. With an identifiable parameter matrix, a numerical nonlinear inductance model is developed, in which the rotor position and the stator current are taken as the variables. After experimentally identifying out all the parameters, a nonlinear mathematic model of SPMSM is built up. With all the machine dimensions and physical parameters, Finite Element Analysis (FEA) is applied to calculate the magnetic field of the machine. FEA and performance simulation results are used to verify the new nonlinear model, which can be further used as a virtual model to develop, simulate and verify the saturation saliency detection strategies.
\end{abstract}

Index Terms - Finite element methods, nonlinear magnetic, PM machines, saturation saliency.

\section{INTRODUCTION}

$\mathrm{T}$ HE permanent magnet (PM) machines, such as permanent magnet synchronous machines (PMSMs), have found wide applications due to their high power density (compactness), high efficiency, ease of control, high torque-to-inertia ratio, and high reliability. However, the rotor position sensor is always required in the PMSM drive systems which will not only increase the system cost, but also perhaps more importantly reduce the system reliability [1]. Even for some instinctive sensorless drive methods such as direct torque control (DTC), the initial rotor position detection is unavoidable [2] [3].

A number of techniques for position sensorless and initial rotor position detection have been proposed based on tracking the rotor magnetic saliency [4] [5]. The rotating magnetic saliencies inside a PMSM can be classified as the structural saliency that mainly comes from the interior structure, and the saturation saliency induced by the magnetic saturation effect of the stator core. In most of the reported sensorless schemes, the structural saliency is employed to determine the position of the rotor axis, while the saturation saliency, which is related to the rotor permanent magnets, can be used to detect the rotor polarity. However, these sensorless strategies can only achieve good performance for interior PMSM (IPMSM) [6] but not for SPMSM, which has little structural saliency and always could be neglected.

On the other hand, the conventional PMSM model does not incorporate the saturation saliency [7]. When developing a new method for the rotor position detection, it is not possible to numerically simulate the proposed scheme, and the experimental trial and error method is widely employed, which is time-consuming and uncertain. Furthermore, there is no scale or a model to evaluate the efficiency and performance of the proposed sensorless schemes.

Digital Object Identifier inserted by IEEE
In this paper, a nonlinear mathematical model of SPMSM is built up considering magnetic saturation saliency. A numerical nonlinear inductance model is proposed based on the stator currents and rotor position variation with all the parameters experimentally identified. FEA is applied based on the machine physical parameters. The magnetic fields of the machine under different currents and variable rotor positions are calculated [8]. The FEA and experiment results are used to verify the machine model. The comprehensive nonlinear machine model is built up in SIMULINK and the performance is simulated. This model can be further used to develop, simulate and evaluate the saturation saliency detection strategies.

\section{NONLINEAR MODEL OF SPMSM WITH SALIENCIES}

In a SPMSM, the observable total flux linkage $\lambda_{t}$ inside the air-gap is contributed by both the stator currents and the permanent magnet on the rotor and it is the link of the stator and the rotor magnetic fields. The three-phase flux linkages $\lambda_{a b c}$ are here defined as the projection of $\lambda_{t}$ on the stator reference frame and not only induced from the stator current. The voltage equation of the stator windings can be written as

$$
v_{a b c}=R_{s} i_{a b c}+\frac{d}{d t} \lambda_{a b c}
$$

where $v_{a b c}, i_{a b c}$ and $R_{s}$ are the phase voltages, currents and winding resistance in the stator reference frame, respectively.

The relationship between current and flux linkage is usually described by the nonlinear magnetization curve. For conventional linear PMSM models, this magnetization curve is always assumed to be linear, and the linear PMSM model is often expressed as

$$
v_{a b c}=R_{s} i_{a b c}+L_{a b c} \frac{d}{d t} i_{a b c}
$$


where $L_{a b c}$ is the three-phase inductance matrix including the self- and mutual-inductances and it is independent of the stator currents. In the rotor reference frame, the $\mathrm{d}$ - and q-axis inductances are thereby constant. When a set of unequal d- and q-inductances is employed, the structural saliency of a PM machine can be calculated based on this model. However, this linear model could not be used to describe the nonlinear saturation saliency.

For a nonlinear model, the magnetic saturation should be considered and the inductances are expressed as incremental inductances variable with the stator current. Then a composite function can be used to express the flux linkage

$$
\lambda_{a}=f\left(i_{a b c}, \theta\right)
$$

where $\theta$ is the rotor position angle.

Substituting (3) into (1), the following differential equations can be obtained

$$
\left[\begin{array}{c}
v_{a} \\
v_{b} \\
v_{c}
\end{array}\right]=R_{s}\left[\begin{array}{c}
i_{a} \\
i_{b} \\
i_{c}
\end{array}\right]+L_{a b c}^{*} \frac{d}{d t}\left[\begin{array}{c}
i_{a} \\
i_{b} \\
i_{c}
\end{array}\right]+\frac{\partial}{\partial \theta}\left[\begin{array}{c}
\lambda_{a} \\
\lambda_{b} \\
\lambda_{c}
\end{array}\right] \omega_{e}
$$

where $\frac{\partial}{\partial \theta}\left[\begin{array}{l}\lambda_{a} \\ \lambda_{b} \\ \lambda_{c}\end{array}\right] \omega_{e}$ is the rotating back-emf in three-phase windings;

$$
\begin{aligned}
& L_{a b c}^{*}=\left[\begin{array}{lll}
\frac{\partial \lambda_{a}}{\partial i_{a}} & \frac{\partial \lambda_{a}}{\partial i_{b}} & \frac{\partial \lambda_{a}}{\partial i_{c}} \\
\frac{\partial \lambda_{b}}{\partial i_{a}} & \frac{\partial \lambda_{b}}{\partial i_{b}} & \frac{\partial \lambda_{b}}{\partial i_{c}} \\
\frac{\partial \lambda_{c}}{\partial i_{a}} & \frac{\partial \lambda_{c}}{\partial i_{b}} & \frac{\partial \lambda_{c}}{\partial i_{c}}
\end{array}\right] \quad \text { is the } \\
& =\left[\begin{array}{lll}
L_{a a}^{*}\left(i_{a b c}, \theta\right) & L_{a b}^{*}\left(i_{a b c}, \theta\right) & L_{a c}^{*}\left(i_{a b c}, \theta\right) \\
L_{b a}^{*}\left(i_{a b c}, \theta\right) & L_{b b}^{*}\left(i_{a b c}, \theta\right) & L_{b c}^{*}\left(i_{a b c}, \theta\right) \\
L_{c a}^{*}\left(i_{a b c}, \theta\right) & L_{c b}^{*}\left(i_{a b c}, \theta\right) & L_{c c}^{*}\left(i_{a b c}, \theta\right)
\end{array}\right]
\end{aligned}
$$

nonlinear inductance matrix which is also a composite function of the stator currents and the rotor position.

\section{NONLINEAR INDUCTANCE MODEL}

\section{A. Nonlinear Mathematical Model}

As shown above, the inductance of the stator is a function of both the stator currents and the rotor position, which are linear independent [9]. For a fixed rotor position, the inductance curve can be expressed as a multinomial

$$
L(i)=l_{0}+l_{1} i^{1}+l_{1} i^{2}+\cdots+l_{m} i^{m}
$$

where $l_{0}, l_{1}, l_{2} \ldots l_{m}$ are the inductance coefficients.

On the other hand, for a constant stator current, the inductance is a periodic function of the rotor position [3]. It can be expressed as the sum of a series of sinusoidal harmonics by using Fourier Series as (6).

$$
L(\theta)=a_{0}+\sum_{n=1}^{\infty}\left(a_{n} \cos (n \theta)+b_{n} \sin (n \theta)\right)
$$

Then a composite function of both the current and rotor position is defined to express the inductance

$$
L(i, \theta)=I(i) \cdot A \cdot C(\theta)
$$

where $I(i)=\left[\begin{array}{lllll}1 & i^{1} & i^{2} & \cdots & i^{m}\end{array}\right]$

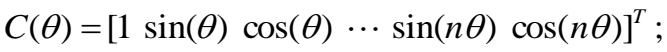

$$
\begin{aligned}
& A=\left[\begin{array}{cccc}
a_{0,0} & a_{0,1} & \cdots & a_{0,2 n} \\
a_{1,0} & a_{1,1} & \cdots & a_{1,2 n} \\
\vdots & \vdots & \ddots & \vdots \\
a_{m, 0} & a_{m, 1} & \cdots & a_{m, 2 n}
\end{array}\right] \text { is the identifiable }
\end{aligned}
$$

parameter matrix.

\section{B. Parameters Identification}

An experiment platform is carried out on an SPMSM to identify the parameters and test the inductances. The block diagram is shown in Fig. 1. During the test, the stator currents are fixed at several different levels from 0 to $6 \mathrm{~A}$ at which the magnetic circuit is fully saturated. For each current offset, by applying a smaller AC current component the incremental inductance of a particular rotor position is measured. By changing the rotor position with a dividing head, a series of inductance is recorded with a resolution of 6 electrical degrees.

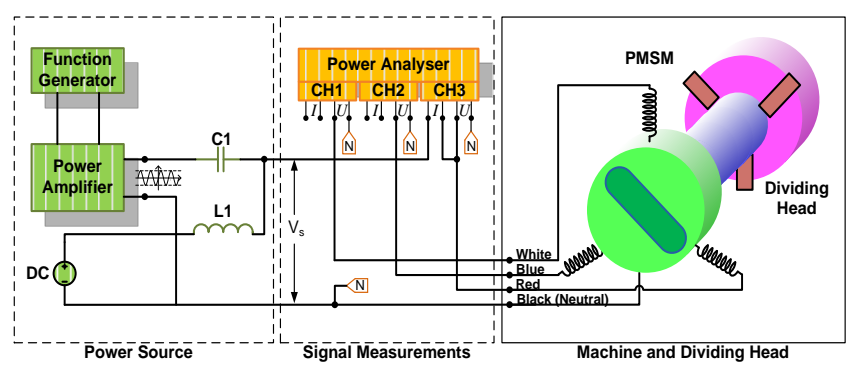

Fig. 1. The experiment platform for inductance test.

The magnetization curve is usually expressed by using a five or seven order multinomial. In order to fit a seventh order saturation curve, $m$ is set to 6 in (5) and (7).

On the other hand, for the periodical inductance variation on rotor position change, the order of Fourier component vector $C(\theta)$ is chosen based on the harmonics weights. Fig. 2 shows the inductance curves at different current offset levels. It is found that the harmonics of the inductance mainly converge in the first 8 orders based on the FFT of the inductances at different currents. In order to regress the inductance-position curve accurately, $\mathrm{n}$ is set to 8 in (7). 
Therefore, the dimension of matrix $\mathrm{A}$ is set to $7 * 17$, by setting $m=6$ and $n=8$ to acquire an accurate enough surface regression.

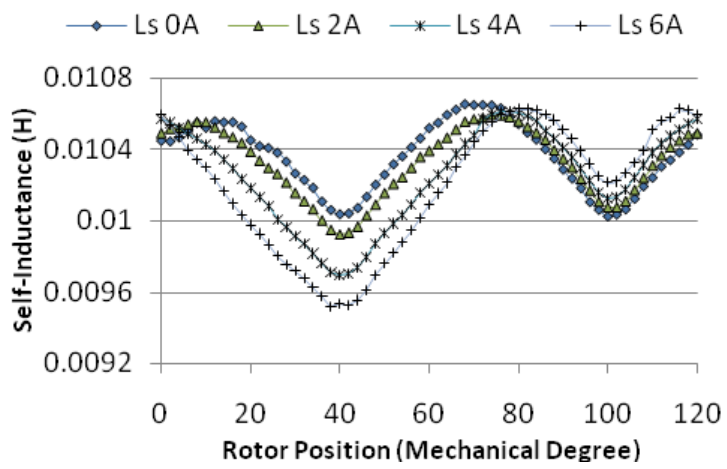

Fig. 2. Measured self-inductance curves at different current offset levels.

The inductances are small and periodical fluctuant function. To obtain better regression results, the Least Relative Residual Sum of Square (LRRSS) method is employed. For the objective function $L(i, \theta)$, the relative residual sum of square is defined as

$$
E_{r e}(A)=\sum_{i=0}^{6} \sum_{\theta=0}^{2 \pi}\left[\frac{I(i) \cdot A \cdot C(\theta)-L_{\text {test }}(i, \theta)}{L_{\text {test }}(i, \theta)}\right]^{2}
$$

Fig. 3 is the comparison between the tested and estimated self-inductance of phase $\mathrm{A}$ at $0 \mathrm{~A}$ and $6 \mathrm{~A}$ current offsets, where $\pm 0.5 \%$ error bands are added. It can be found that the relative errors of the inductances are very small and the regressed objective function can be used to describe the variable selfinductance. The same regression method is applied to the mutual-inductance coefficients identification. Then a nonlinear inductance model is built up for this three-phase machine. An accurate inductance matrix can be calculated for given stator currents and rotor position. This model incorporates both the machine structural and the saturation saliencies.

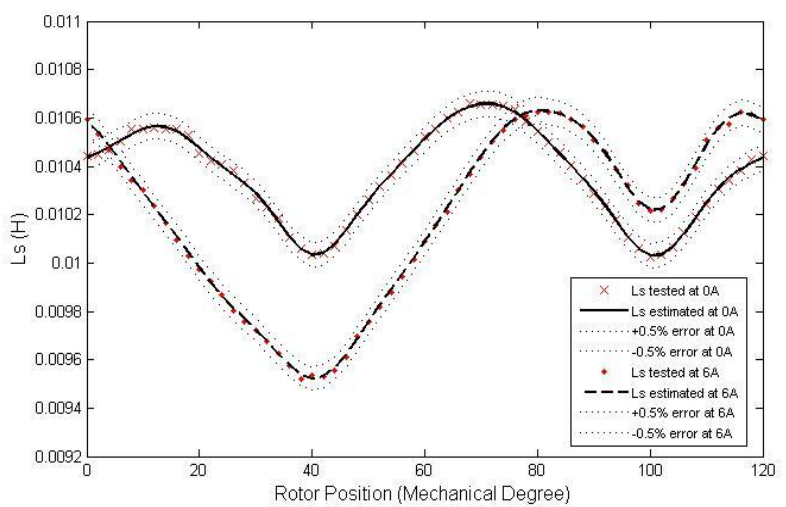

Fig. 3. Measured and estimated self-inductance at different current offsets.

\section{Model VerificATION BY FEA}

To verify the new nonlinear inductance model, the 2dimensional FEA method is applied to numerically calculate the magnetic field of the machine. The data of the machine physical parameters is collected, including the material characteristics of the magnets and the core. After dismantling the machine, the inner dimensions are also measured. Fig. 4 is the comparison of estimated and calculated inductances.

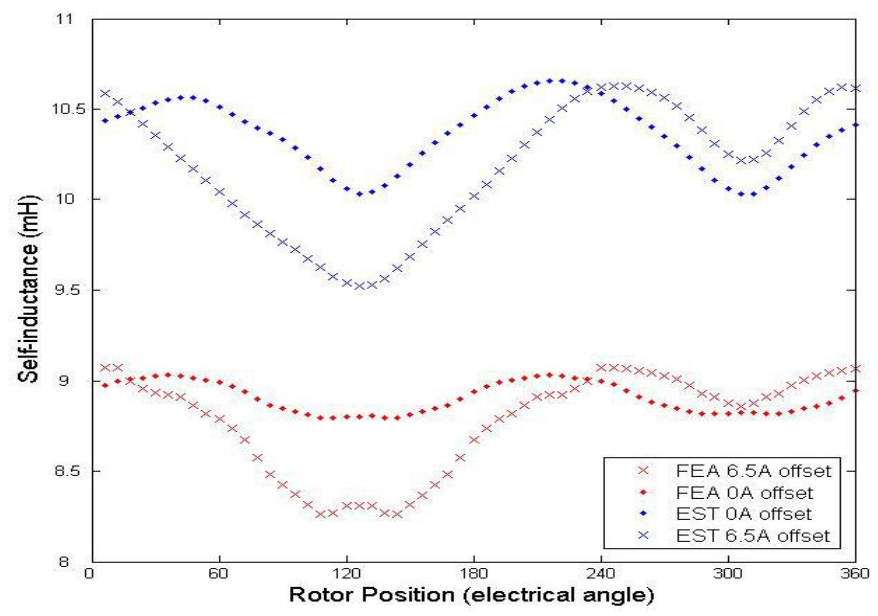

Fig. 4. Comparison of the estimated and calculated self-inductances.

It can be found that the calculated inductances coincide with the estimated values. The errors shown in Fig. 4 mainly come from the dimension measurements and the stator skewed slots. The ending leakage inductances of the stator windings and the skewed slots on the stator are not considered in this 2dimensional FEA algorithm. The stator leakage inductance is calculated separately and the value is about $1.9 \mathrm{mH}$.

\section{PERFORMANCE}

A comprehensive nonlinear three-phase inductances model is built up in SIMULINK. This new model is different from the linear machine model given in the SIMULINK library.

Three-phase sinusoidal stator currents are input to the model with the synchronous rotor position. When it is operating at no-load or a light load, the current amplitude is small and there is no saturation in the stator core. The inductance curves are unsaturated as shown in Fig. 5. It is also can be found that the structural saliency of this machine is very small.

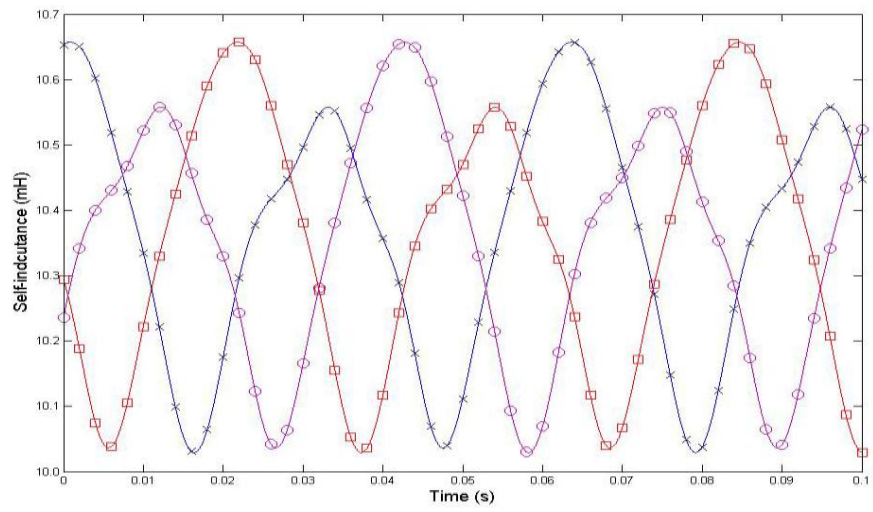

Fig. 5. Unsaturated three-phase self-inductance curves at no-load.

Then, a larger current vector is input to the stator windings at which the stator core needs to be fully saturated. Based on the 
experiment results of the inductance tests, the currents are set at $6.5 \mathrm{~A}$ and the saturated inductance curves are shown in Fig. 6 . It can be found that the saturation effect changes the inductance profile and the period of the phase self-inductance is $2 \pi$, in which the rotor polarity is clearly involved.

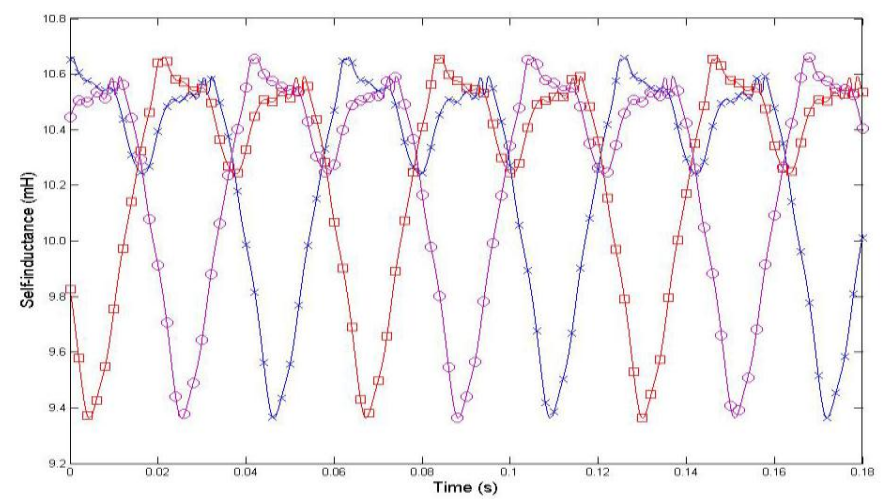

Fig. 6. Saturated three-phase self-inductance curves at $6.5 \mathrm{~A}$ stator current.

Based on this new nonlinear inductance saturation model, the mathematical model of the SPMSM is built in MATLAB/SIMULINK, in which both the structural saliency and the saturation saliency are considered.

The new machine model is simulated with open loop drive method. It is started without load and then a load torque is mounted on the rotor shaft, at which the three-phase stator inductances are saturated. The machine speed and torque output curves are shown in Fig. 7.
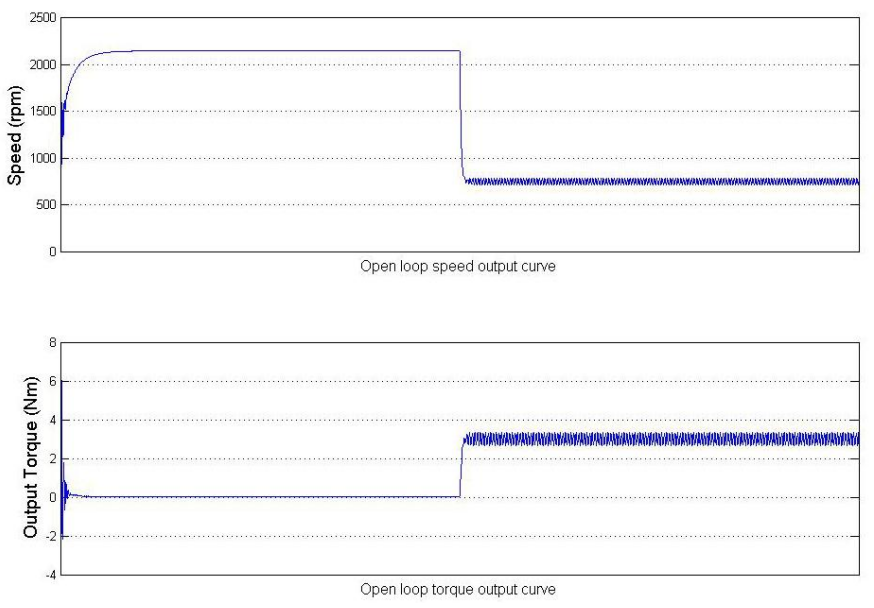

Fig. 7. Speed and torque output of open loop operation with a load torque step.

It can be found that when the machine running at no-load the output torque is stable because the three-phase inductances are unsaturated and the equivalent $\mathrm{d}$ - and $\mathrm{q}$-axis inductances are almost constant which induce a constant torque output on the shaft.

On the other hand, after the load torque stepping up, the stator currents rise up and the stator core is saturated. There are ripples on the speed and torque output caused by the saturated and asymmetry inductances. These features make the simulation more accurate, but do not exist in the linear machine model simulations.

\section{CONCLUSION}

The nonlinearity of the PMSM machine model is mainly caused by the saturable inductances of the stator windings. In this paper, a composite function is designed to express the inductance as the function of both the stator current and the rotor position which are de-coupled. A nonlinear model of PMSM is setup based on this function.

An experimental platform is carried out to identify the inductance coefficients. After taking a set of inductance measurements, the self- and mutual-inductances at different current level and different rotor positions are recorded. The LRRSS method is employed and the regression results are verified by FEA calculation. Based on the FEA results, the nonlinear inductance model is reasonable and effective to simulate the real machine.

With the identified coefficients, the new SPMSM model is built up in MATLAB/SIMULINK. The unsaturated and the saturated inductance curves are simulated and the no-load operation simulation of the new model is also carried out. The effect of the saturation saliency can be found from the simulation results.

Based on this new comprehensive model, simulation of the new proposed novel drive methods will be possible which will avoid the experimental trial and error process, reduce the develop cycle time and save the research costs.

\section{REFERENCES}

[1] Y. Yamamoto, Y. Yoshida and T. Ashikaga, "Sensorless control of PM motor using full order flux observer", IEEE Transactions on Industrial Applications, Vol. 124, No. 8, pp.743-749, Aug. 2004.

[2] M. E. Haque, L. Zhong and M. F. Rahman, "A sensorless initial rotor position estimation scheme for a direct torque controlled interior permanent magnet synchronous motor drive", IEEE Transactions on Power Electronics, Vol. 18, No. 6, pp.1376 - 1383, Nov.2003.

[3] L. Tang, L. Zhong, M. F. Rahman and Y. Hu, "A novel direct torque control for interior permanent magnet synchronous machine drive system with low ripple in torque and flux-a speed sensorless approach", in Proceedings of the Industry Applications Conference, Oct. 2002, Vol. 1, pp.104- 111

[4] E. Robeischl, M. Schroedl and M. Krammer, "Position-sensorless biaxial position control with industrial PM motor drives based on INFORM-method and back EMF model", in Proceedings of the IEEEIES Annual Conference, 5-8 Nov. 2002, Vol. 1, pp. 668 - 67.

[5] S. Kondo, A. Takahashi and T. Nishida, "Armature current locus-based estimation method of rotor position of permanent magnet synchronous motor without mechanical sensor", in Proceedings of IEEE-IAS Annual Meeting, 1995, Vol. 1, pp.55-60.

[6] Y. Jeong, R. D. Lorenz, T. M. Jahns, and S. K. Sul, "Initial rotor position estimation of an interior permanent-magnet synchronous machine using carrier-frequency injection methods," IEEE Transactions on Industrial Applications, vol. 41, no. 1, pp. 38-45, Jan./Feb. 2005.

[7] Y. Yan, J. G. Zhu, Y. Guo and H. Lu, "Modeling and simulation of direct torque controlled PMSM drive system incorporating structural and saturation saliencies," in Proceedings of the $41^{\text {st }}$ IEEE Industry Application Society Annual Meeting, Tampa, FL, Oct 2006, pp. 76-83.

[8] Shuhong Wang, Jie Qiu, Qingfu Li, Jian Guo Zhu and Semyung Wang, "Application of Petri Net in Development of Finite Element Analysis Package for Electromagnetic Fields", IEEE Transactions on Magnetics, vol. 42, no. 4, pp. 1255-1258, 2006

[9] P. Cui, J. G. Zhu, Q. P. Ha, G. P. Hunter, and V. S. Ramsden, "Simulation of non-linear switched reluctance motor drive with PSIM," in Proceedings of the $5^{\text {th }}$ International Conference on Electrical Machines and Systems, Vol. 1, Aug. 2001, pp. 1061-1064. 